\title{
CLIMATE CHANGE INDUCED FLOOD RISK AND ADAPTATION IN THE PADMA RIVER ISLAND, BANGLADESH: A LOCAL SCALE APPROACH
}

\author{
${ }^{*}$ Rumana Sultana ${ }^{1}$, S. Rafiqul Alam Rumi ${ }^{2}$ and M. A. Hanif Sheikh ${ }^{3}$
}

\author{
${ }^{1}$ Corresponding Author ${ }^{*}$, Ph D Fellow, Department of Environment and Energy System, Graduate School of \\ Science and Technology, Shizuoka University, Japan, Email: rumanaslt@gmail.com \\ ${ }^{2,3}$ Professor, Department of Geography and Environmental Studies, University of Rajshahi, Bangladesh
}

\begin{abstract}
Assessment of hazard characteristics, exposure, vulnerability, resilience and risk at a local scale is an important tool for identifying the capacities of households and local communities to adapt with climate change induced flood. Repeated floods with different magnitudes under variable climatic conditions are great catastrophe for the people living in flood prone regions specifically in Islands. People, infrastructure and economy are highly exposed to flood and that results in devastating flood disaster. In the Padma river Islands frequent flooding makes the life and livelihoods of people vulnerable and this vulnerability is conditioned by different factors such as people's physical, social, economic and environmental condition. The higher is the level of vulnerability the higher is the level of flood risk. Hence, people's resilience can minimize the negative impacts of flood disaster. A composite risk index (related to the probability of occurrence of the event, magnitude of the event, quantity and cost of the element at risk, vulnerability and resilience) was prepared to realize the climate change induced flood disaster risk. Some adaptive strategies are explored to adapt with climate change induced flood risk. Finally, Local Scale Disaster Risk Model (LSDR) was proposed to present the framework of climate change induced flood disaster risk and adaption at a local scale.
\end{abstract}

Keywords: Climate change, exposure, flood, rainfall, risk, resilience and vulnerability.

সারাংশ: জলবায়ু পরিবর্তন ত্রান্বিত বন্যার সাথে বসতি ও সম্প্রদায়ের অভিযোজনের সক্ষমতা নির্ণয়য়ের জন্য অত্যন্ত গুরুত্বপূর্ণ পদ্ধতি হচ্ছে স্থানীয় পর্যায়ে দুর্যোগের বৈশিষ্ট্য, এক্সপোসার, ভালনেরাবিলিটি, রেসিলিয়েন্স এবং রিস্ক নিরূপণ। পরিবর্তনশীল জলবায়ুতে পুনঃ পুনঃ সংঘটিত বন্যা সংকটাপন্ন এলাকায় বসবাসরত মানুষের জন্য বিশাল দুর্ভোগ, বিলেষ করে চরবাসীর জন্য। জনগণ, অবকাঠানো এবং অর্থনীতি বন্যার দ্বারা অতিমাত্রায় ক্ষতিগ্রস্ত হয়ে ভয়ঙ্করী দুর্যোগের সৃষ্টি করে। পদ্মানদীর চরে পুনঃ পুনঃ সংঘটিত বন্যা মানুষের জীবন ও জীবিকা প্রতিপন্ন করে তোলে এবং এই প্রতিপন্নতার নিয়ামকগুলো হচ্ছে অবকাঠানো, সামাজিক, অর্থনৈতিক ও পারিবেশিক অবস্থা। প্রতিপন্নতার মাত্রা যত বেশি হয়, রিস্ক এর মাত্রাও ঠিক ততটাই বেশি হয়। তবে, মানুযের রেসিলিয়েন্স বন্যার নেতিবাচকে দিকগুনো প্রশমন করতে পারে। জলবায়ু পরিবর্তন ত্বরান্বিত বন্যার দুর্যোগ রিস্ক নিরূপণের জন্যএকটি সম্মিলিত রিস্ক ইনডেক্স (যা ঘটনা সংঘটনের সম্ভাবনা, ঘটনার মাত্রা, সংকটাপন্ন উপাদানের পরিমাণ ও মূল্য, ভালনেরাবিলিটি এবং রেসিলিয়েন্স এর সাথে সমপর্কিত) টৈরি করা হয়েছে। বন্যার সাথে অভিযোজনের জন্য কিছু কৌশল উন্নোচন করা হয়েছে। সর্বশেযে, স্থানীয় পর্যায়ে জলবায়ু পরিবর্তনজনিত ত্বরান্বিত বন্যার দুর্যোগ রিস্ক এবং অভিযোজন এর কাঠামোস্বরূপ লোকাল স্কেল ডিসাসটার রিস্ক মডেল (এলএসডিআর) প্রস্তাব করা হয়েছে।

\section{Introduction}

Bangladesh is a hotspot of risk from extreme weather events as well as flood disaster and level of risk in many islands (Such as Narayanpur, Purba khas Bandarkhola etc) is likely to grow due to combination of rising rainfall and repeated flood events. Rapid change in global temperature caused fundamental change on our climate like increasing pre monsoon rain. Extreme weather and climatic events, interacting with exposed and vulnerable people and natural systems, can lead to disasters (IPCC, 2012). Catastrophic floods have intensified and taking place more frequently owing to increased rainfall in monsoon. Over the last twenty years, Bangladesh has been ravaged floods of devastating proportion in 1998, 1999, 2004, and 2007 (UNDP, DFID, 2007). In the present study area up warding tendency of pre monsoon rain was seen during the year 1998 and 2007 and it results in catastrophic flood there. Climate change induced flood produce widespread damage and several alterations in the normal functioning of communities. Bangladesh is recognized worldwide as one of the most vulnerable countries to the negative impacts of climate change related disasters. High level of exposure, hazard magnitude, vulnerability and low level of resilience makes normal flood devastating in the Padma river Island (for example: Narayanpur Island). The people and society have knowledge and experience of coping with the impact to some degree and extent.

\section{Conceptual Framework for a Local Scale Risk Indicator System}

A local scale risk indicator system is developed to manage climate change flood disaster risk at community level. Risk indicator system is a useful tool for measuring risk numerically by selecting several indicators related to the main factors (Table: 1). The following conceptual framework defines the key elements of risk management into the factors of hazard, exposure, vulnerability and resilience. The factors help to identify the appropriate indicators. This system helps to conduct a research more quickly and to present the final result more easily for the reader (specialist and common people). Selection of the indicator is an important factor here because wrong selection of indicator can give unrealistic result. The resulting indicator system comprises a total of 31 indicators, arranged according to the identified four main factors and further broken down into factor components. 


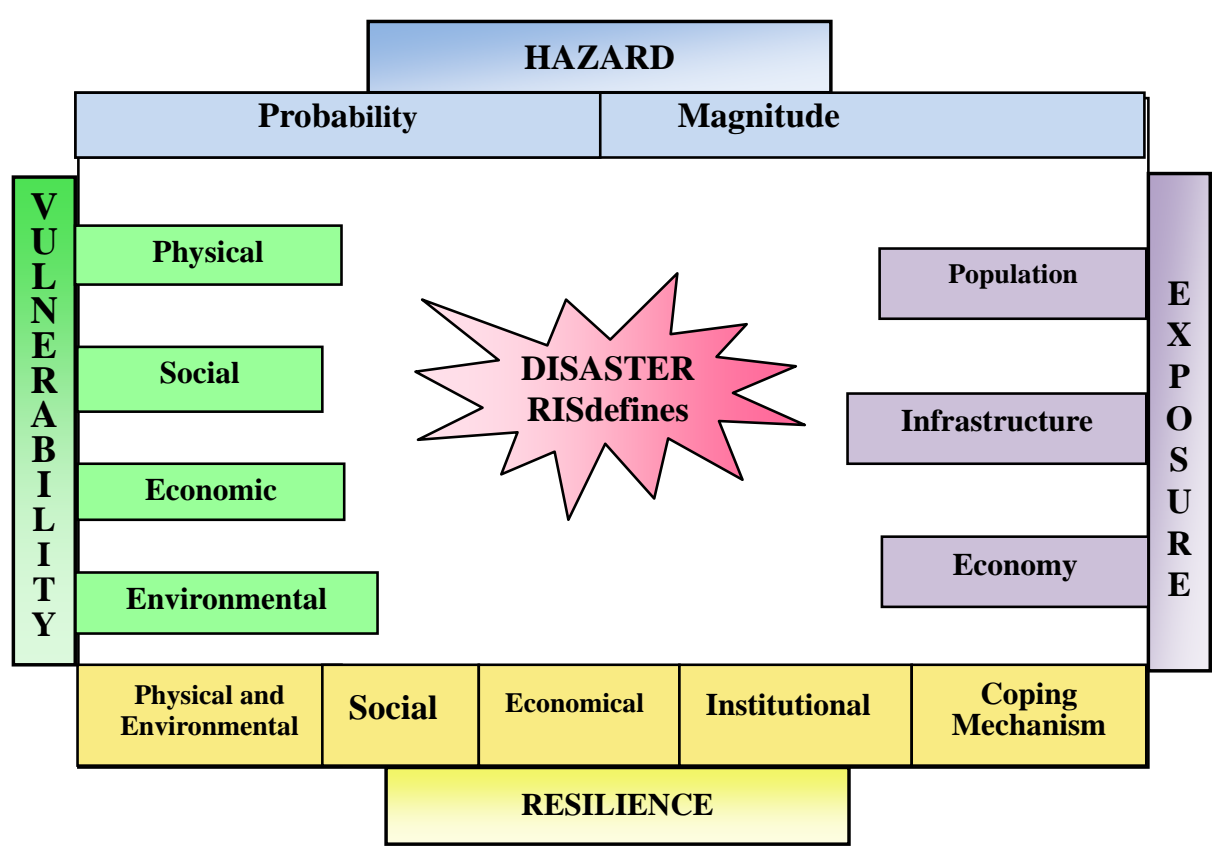

Fig. 1 Conceptual framework for a local scale disaster risk indicator system

This indicator system is expected to developing the capacity of decision makers at local level to measure key elements of climate change induced disaster risk and vulnerabilities for communities.

This concept helps to provide comparative parameters for monitoring changes in climatic variables and disaster risk formation.

\section{Preparation for Local Scale Risk Index}

This indicator system summarizes a great deal of information in a simple way using factor scores as well as factor components. This provides good insights of the impact of climate change and adaptation with regard to the factors that determine risk.

In first step, the different measurement of the individual indicators has to be made comparable through scaling. This is done by assigning a value of 1, 2, 3, 4 or 5 (very low, low, medium, high, very high). Depending on the scaled indicator values, the factor scores vary between 0 to 100 . This can be prepared by distributing a total of 33 weightening points according to the believed importance of the indicators for each factors. Importance of different indicators is different.

The table 1 represents four factors of this research and associated indicators of those factors. Indicators were selected based on literatures, expert opinion and pilot survey in the study area to get dataset related to the factors associated with risk. Data regarding the indicators was collected from primary and secondary sources.

\section{Rainfall and Flood Events}

Climate change is triggering the recent heavy rain and flooding along the river Padma (Figure: 2). More intense precipitation causes extreme flood events in the Padma river island. Analyzed Rainfall data collected from Rajshahi Station, the nearest station from the study area (Narayanpur Mauza), shows that after the year 1990 frequency of extreme precipitation has increased. The year 2007 represents the highest average rainfall value of more than $400 \mathrm{~mm}$. As monsoon rainfall is increasing in future the average monsoon rainfall will become much more than that of present (Figure: 2). The linear trend line of average monsoon rainfall represents an upwarding trend. In this study, the secondary data from the station nearest to the present study area was analyzed to understand local disaster risk.

Figure 2 shows that there is a upwarding trend in average pre monsoon rainfall at Rajshahi station.

Between the year 1975 to 1985 there is higher level of precipitation at Rajshahi station. Although annual average rainfall represents a down warding trend, monsson rain fall causes trigger events (flood). From the Focus Group Discussion (FGD) data it is clear that 
Table 1 Factor components and indicators

\begin{tabular}{|c|c|c|}
\hline $\begin{array}{l}\text { Factor } \\
\text { component } \\
\end{array}$ & Indicator name & Indicator \\
\hline \multicolumn{3}{|l|}{ Flood Hazard } \\
\hline \multirow[t]{2}{*}{ Probability } & (H1)Magnitude & Water discharge in the past 51 years and peoples experience \\
\hline & (H2)Probability & Flood frequency \\
\hline \multirow[t]{3}{*}{ Magnitude } & (H3)Rainfall & Pre-monsoon, Monsoon and annual average \\
\hline & (H4)Water level & Water level cross the danger level. \\
\hline & (H5)Duration & People's perception on flood Duration \\
\hline \multicolumn{3}{|l|}{ Exposure } \\
\hline Population & (E1)people at risk & $\%$ of affected population( every year) \\
\hline Structure & $\begin{array}{l}\text { (E2)Construction material of house } \\
\text { (E3)Infrastructure at risk }\end{array}$ & $\begin{array}{l}\% \text { of house made of flood resilient construction material } \\
\text { money value of infrastructure at risk }\end{array}$ \\
\hline \multirow[t]{2}{*}{ Economy } & (E4)Household content at risk & Money value of household content at risk \\
\hline & (E5)Outside properties a risk & Money value of outside properties at risk \\
\hline \multicolumn{3}{|l|}{ Vulnerability } \\
\hline \multirow[t]{2}{*}{ Physical } & (V1)Flood prone settlements & $\%$ of settlement in flood prone area \\
\hline & (V2)Unprotected settlement & Proportion buildings go under water \\
\hline \multirow{4}{*}{ Social } & (V3)Literacy rate & Level of education \\
\hline & (V4)Access to basic needs & $\%$ of people have access to basic needs \\
\hline & (V5)Household size & Members in a household \\
\hline & (v6)Medical service & Time Distance from house to \\
\hline $\begin{array}{l}\text { Factor } \\
\text { component }\end{array}$ & Indicator name & Indicator \\
\hline \multirow[t]{3}{*}{ Economic } & (V7)Income & level of income \\
\hline & (V8)Diversification & $\%$ of population \\
\hline & (V9)Savings & savings in TK \\
\hline \multirow[t]{2}{*}{ Environmental } & (V10)Green space & Proportion of green space per household \\
\hline & (V11)Land erosion & \%of population suffer from land erosion \\
\hline \multicolumn{3}{|r|}{ P P P } \\
\hline \multirow{4}{*}{$\begin{array}{l}\text { Physical and } \\
\text { environmental } \\
\text { Social }\end{array}$} & $\begin{array}{l}\text { (R1)Location } \\
\text { (R2) Environmental resource }\end{array}$ & $\begin{array}{l}\text { \% of people have access to safe location } \\
\text { available environmental resources }\end{array}$ \\
\hline & (R3)Age & Age of the respondent \\
\hline & (R4)Emergency groups & People get assistance from emergency group \\
\hline & $\begin{array}{l}\text { (R5)Awareness and Capacity building } \\
\text { programmes }\end{array}$ & Frequency of awareness building programmes \\
\hline Economic & $\begin{array}{l}\text { (R6)Access to Livelihood assets } \\
\text { (R7)Access to national and international } \\
\text { emergency fund }\end{array}$ & $\begin{array}{l}\text { Access to Physical, social, economic, environmental and financial } \\
\text { assests Employability, good health and knowledge Peoples access to } \\
\text { emergency fund }\end{array}$ \\
\hline Institutional & $\begin{array}{l}\text { (R8)Early warning system } \\
\text { (R9)Interaction }\end{array}$ & $\begin{array}{l}\% \text { of population get early warning } \\
\text { level risk management institution }\end{array}$ \\
\hline $\begin{array}{l}\text { community coping } \\
\text { mechanism }\end{array}$ & (R10) Coping strategies & Economic, Social and Technological/structural coping strategies \\
\hline \multicolumn{3}{|c|}{ Finally the formula given below is used to prepare composite risk index } \\
\hline \multicolumn{3}{|c|}{ Risk = hazard + Exposure + Vulnerability - Resilience (modified after Bollin et al., 2003) } \\
\hline
\end{tabular}

catastrophic flood took place in the present study area during monsoon and the flood has become more frequent and catastrophic after 1990. On the other hand analyzed water level data represents that during the year 1960 to 2012 water level cross the danger level for 21 times.

In Narayanpur island normal flood become catastrophic because of higher vulnerability and lower resilience. According to analyzed Focus Group Discussion (FGD) data, equal to or above17.25 m-PWD level of the Ganges water causes devastating flood here. So, during the last 52 years extreme flood causes in Narayanpur mauza for 48 times.

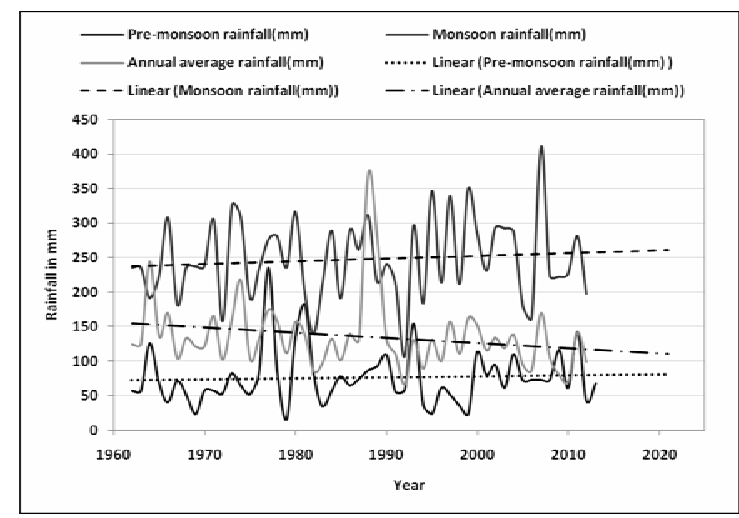

Fig. 2 Rainfall at Rajshahi Station (1962-2012)

Source: Bangladesh Water Development Board (BWDB) 


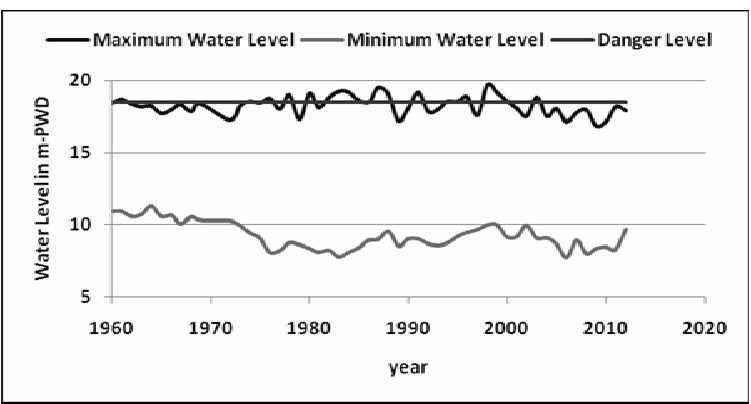

Fig. 3 Rainfall at Rajshahi Station (1962-2012),

Source: Bangladesh Water Development Board (BWDB)

Table 2 Flood Frequency of the river Ganges, 1960-2010

\begin{tabular}{|c|c|c|c|}
\hline $\begin{array}{l}\text { Flood } \\
\text { year }\end{array}$ & Flood type & $\begin{array}{l}\text { Water discharge class } \\
\text { cumec }\end{array}$ & $\begin{array}{l}\text { Flood } \\
\text { Frequency }\end{array}$ \\
\hline 7 & Normal Flood & $30001-40000$ & $1-1.0001$ \\
\hline 13 & Moderate Flood & $40001-50000$ & $1.001-1.08$ \\
\hline 22 & Havoc Flood & $50001-60000$ & $1.13-2.01$ \\
\hline 4 & $\begin{array}{l}\text { Great Havoc } \\
\text { Flood }\end{array}$ & $60001-70000$ & $2.19-4.23$ \\
\hline 5 & $\begin{array}{l}\text { Very Vulnerable } \\
\text { flood }\end{array}$ & $>70000$ & $>4.23$ \\
\hline Mean & 2.70 & & \\
\hline \multicolumn{4}{|c|}{ Criteria of Assessment } \\
\hline 1 & 2 & 4 & 5 \\
\hline Normal & Moderate & Havoc & Very \\
\hline Flood & Flood & $\begin{array}{l}\text { Havoc } \\
\text { Flood }\end{array}$ & $\begin{array}{l}\text { Vulnerable } \\
\text { flood }\end{array}$ \\
\hline
\end{tabular}

Source: Bangladesh Water Development Board (BWDB)

Flood frequency was analyzed using Gumbel,s method. This method of flood frequency analysis involves the fitting of a probability model to the sample of annual flood peaks recorded over a period of observation, for a catchment of a given region. This method is more suitable because it is a stochastic generating structure that produces random outcomes (Mujere, 2011). Discharge data from Hardinge Bridge station, only station to measure Ganges discharge, were used for analyzing. Analyzed flood frequencies are categorized into five types. The peak water discharge of the river Ganges has been classified in the discharge range of 30001-40000cumec that has been called as Normal Flood and the frequency has been observed 1-1.0001 and that has been occurred seven times between the year 1960 to 2010. The second classified range is 40001-50000cumec that has been called moderate flood and the frequency has been observed 1.001-1.08 The next classification is called havoc flood and the discharge ranges from 50001-60000cumec. The frequency of this range is 1.13-2.01. In Gangetic delta this type of flood occurred twenty two times between the years 1960 to 2010. The peak water discharge in the river Ganges has been classified into the discharge range of 60001-70000cumec and this flood type has been called Great Havoc Flood and the flood frequency of this type of flood is observed (table 2) 2.19-4.23.
Great Havoc flood occurred four times in the Gangetic delta, Bangladesh. The peak discharge in the river Ganges has been classified in the discharge range of $>70000$ cumec that has been called as Very Vulnerable Flood and flood frequency has been observed $>4.23$ and this type of flood has been observed five times in the present study area.

\section{Exposure}

Exposure refers to the Element at risk and inventory of those people or artefacts that are exposed to a hazard" (UNDP-BCPR, 2004). In the present study area almost all the people are affected by flood hazard. Low resilient construction material of their houses enhances flood exposure. Most of the houses are made of mud and sand.
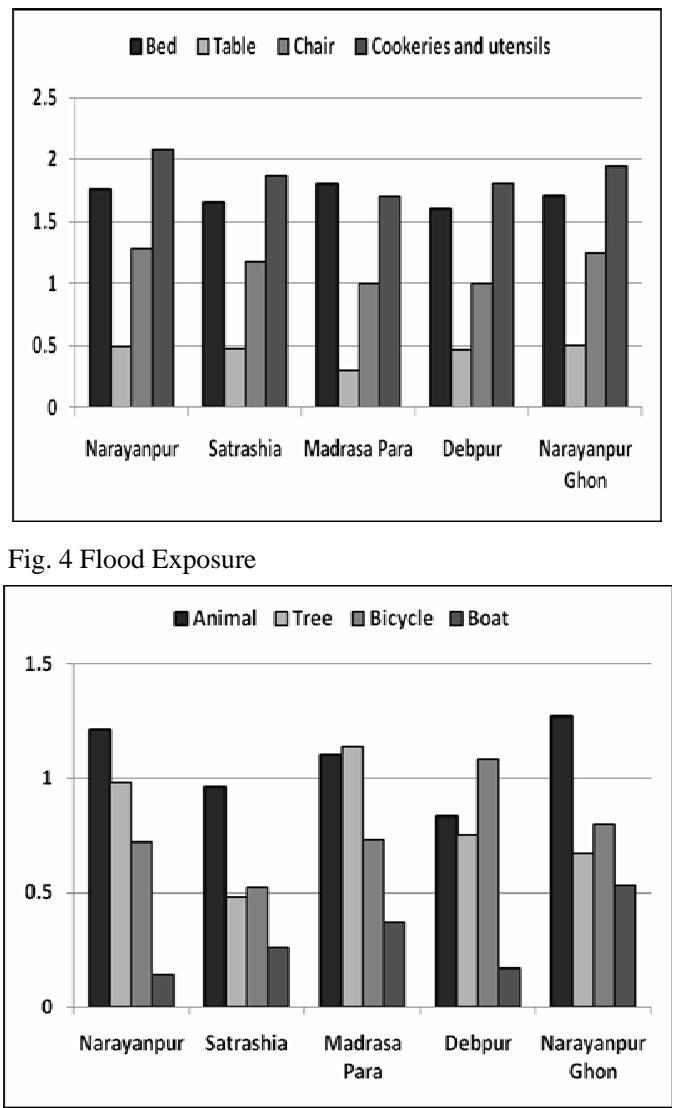

Fig. 4 Mean Index of household contents and outside properties at risk

Source: Field Survey, 2012

Based on observation and questionnaire survey data, in Narayanpur mauza more than half of the respondents think that more than $80 \%$ of the infrastructures are exposed to flood. Here, house hold contents and outside properties are also highly exposed to flood hazard. 


\section{Assessing Vulnerability and Resilience}

IPCC (Intergovernmental Panel on Climate Change) defined vulnerability as "The propensity or predisposition to be adversely affected" (IPCC 2012). In the present study area flood proneness of the settlement is very high. Almost three fifth of the respondents said that once in every year their settlements are affected by flood hazard. More than three fifth of the respondents said that during flood half portion of their buildings usually goes under water. Analyzed data shows that in Narayanpur Mauza, two fifth of the surveyed households have more than nine family members. The larger the household size, the lower the social status and the higher the amount of people affected (Haki et al., 2004 and Cutter et al. 2003). In Narayanpur mauza, less than one percent of the respondents can reach medical within one hour. On the contrary, more than half of the respondents have to

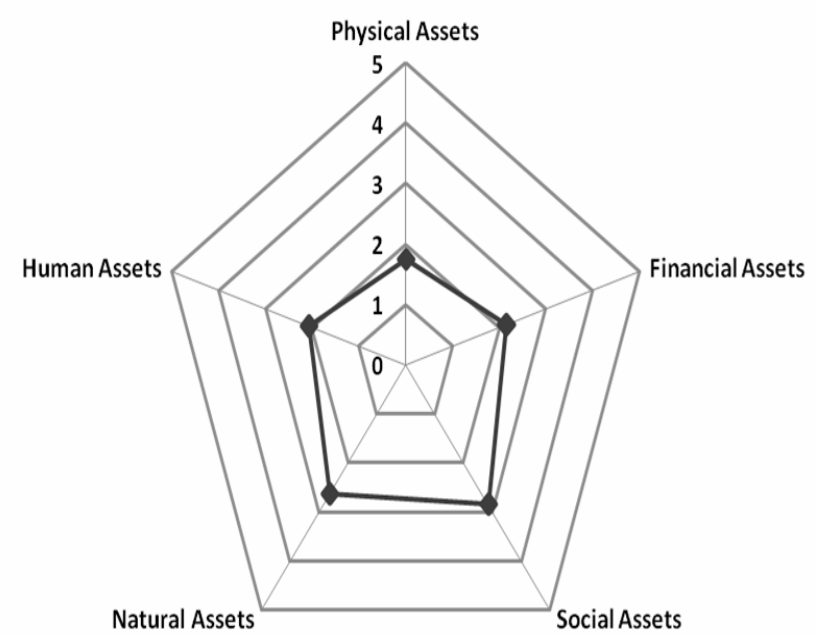

\begin{tabular}{|l|l|l|l|l|}
\hline \multicolumn{5}{|l|}{ Criteria of assessment } \\
\hline $\begin{array}{l}\text { Very } \\
\text { low }\end{array}$ & Low & Moderate & High & $\begin{array}{l}\text { Very } \\
\text { high }\end{array}$ \\
\hline $0-1$ & $1-2$ & $2-3$ & $3-4$ & $4-5$ \\
\hline
\end{tabular}

Fig. 5 Assessing livelihood assets (left) and Mapping Vulnerability (right) Source: Field Survey: 2012 and 2013

Livelihood asset is an important indicator of resilience. The higher is the access to livelihood assets the higher is the resilience. The pentagonal presentation (Figure 3) represents the overall condition of livelihood assets in Narayanpur mauza. Social assets represents the highest and natural assets the second highest grand mean value. Grand mean means mean of the means. On the other hand, physical assets show the lowest grand mean value. The more the access to livelihood assets the spend more than five hours to reach in medical centre or health care centre. Here, level of income of the local people is very low. More than two third of the respondents earn less than 200 taka. Similarly, their income source is not enough diversified to cope with the climate change induces floods. Vulnerability map shows that southern and western parts of the present study area are very highly vulnerable to flood Because of their location in an area that is surrounded by India and has no suitable means of transportation. On the other hand, very small portion in the middle part is least vulnerable. In Narayanpur mauza mauza local people becomes more vulnerable due to unsafe and unprotected location. Assistance from emergency group and frequent awareness building program helps to cnhance the level of resilience and decrease vulnerability.

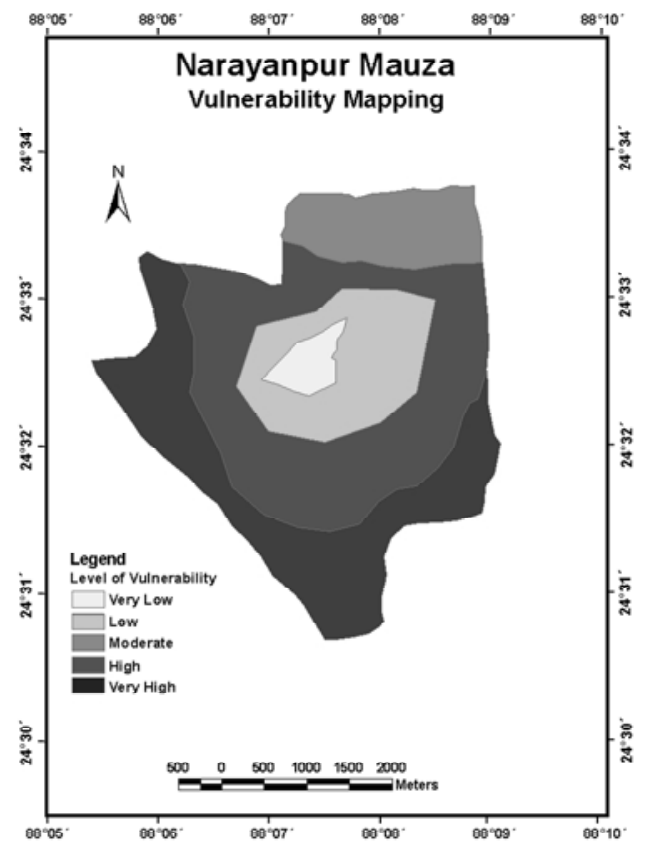

higher is the level of resilience in relation to natural disaster (e.g. flood). In the present study peoples' access to financial, social, natural and human assets is moderate. Peoples' access to physical assets is low there. In Narayanpur Mauza the level of community resilience is not high enough to cope with repeated flood hazard. Lower resiliency increases the negative impacts of climate change induced flood hazard and makes people more vulnerable to flood. 


\section{The Risk Index}

Figure: 4 shows the composite flood risk index Risk = Hazard + Exposure + Vulnerability - Resilience, modified after Bollin et al., 2003) and the factor scores of the community in Narayanpur mauza. The score of hazard is fairly high because of changing rainfall and flooding patterns. The score of vulnerability represents the second highest value due to existing physical, social, economic and environmental vulnerability. The money value of household and outside properties at risk

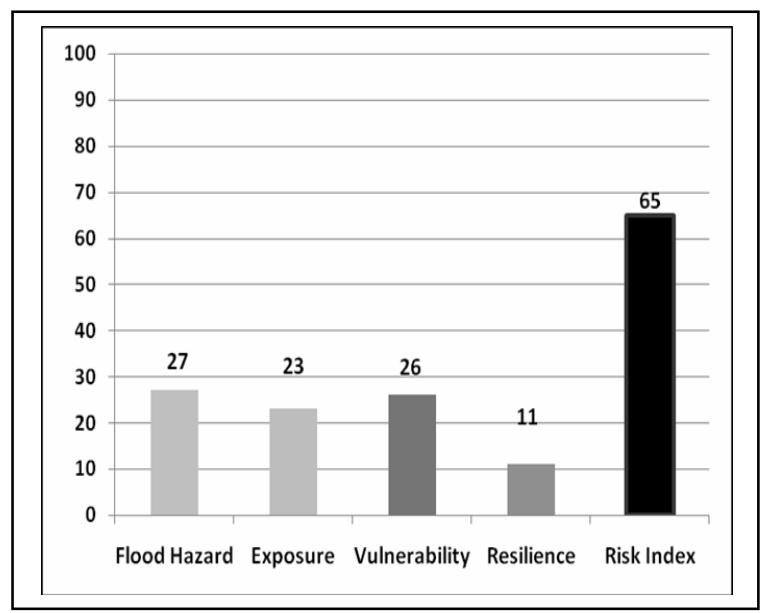

Fig. 6 Composite risk index (left) and comparative risk index (right) Source: Field Survey, 2012

Narayanpur mauza consists of five villages (Narayanpur Ghon, Debpur, Madrasa Para, Satrashia and Narayanpur). Five separate risk index were made for each villages as there were some locational and structural differences among the villages. Comparative risk index shows that risk is highest in the village Satrashia because of high vulnerability and lower resilience. Other reason is that, this village is surrounded by India in three sides and cannot move easily during disaster and also face problem because of ill transportation system. Similarly, village Debpur represents the second highest value. On the other side, risk is lowest in village Narayanpur among the villages in Narayanpur mauza.

\section{Adaptation Strategies}

Different types of coping strategies which can be used in different period of disaster (Pre disaster, During disaster and Post disaster) at local scale to adapt with climate change induced flood. Coping strategies means

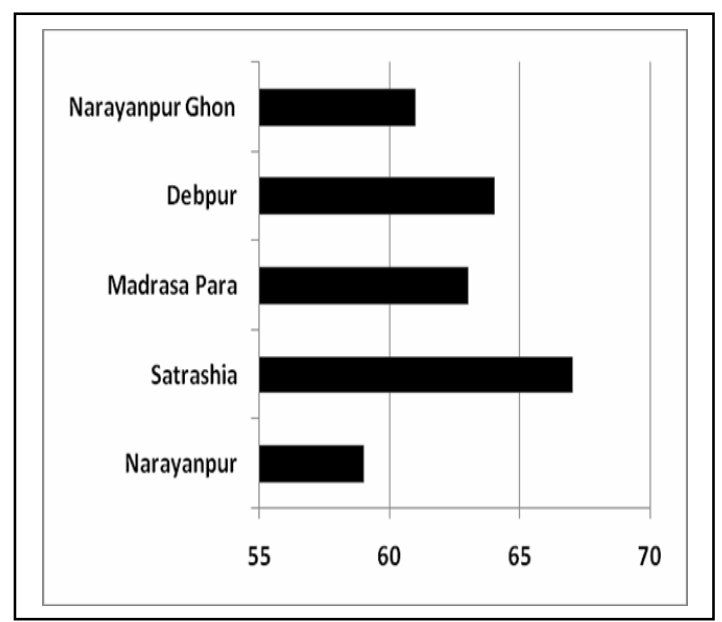

is not high at all. The resilience score is very low because of lack of physical and environmental resilience, social resilience, economic and institutional resilience. Overall risk index represents the climate change flood hazard risk of the community in Narayanpur mauza. The risk is higher because of higher score of flood hazard, exposure and vulnerability. On the other hand, low score of resilience is responsible for risk.

the strategies taken to cope with disaster. In pre disaster period or before disaster strikes peoples can take some strategies like saving money, increasing the height of the base of the house, evacuating important element to safe place etc to become more resilient. This stage is very important to pass a disaster with least negative impact. Using coping strategies during disaster can also minimize the negative impacts. In post disaster period or after passing disaster people sell their important assets or diversify their income source to overcome the income shocks. But taking strategies in this period can be beneficial in short term recovery but not in long term. Most of the economic coping strategies are used in post disaster period. Social coping strategies are used in the three periods of disaster management cycle. Almost all the structural or technological coping strategies are applied in pre disaster period as a preparation to stand against flood before it strikes. 
Table 3: Period of use of coping mechanism

\begin{tabular}{|c|c|c|c|}
\hline \multirow[t]{2}{*}{ Coping Mechanism } & \multicolumn{3}{|c|}{ Period Of Use } \\
\hline & Pre Disaster & $\begin{array}{c}\text { During } \\
\text { Disaster }\end{array}$ & $\begin{array}{c}\text { Post } \\
\text { Disaster }\end{array}$ \\
\hline \multicolumn{4}{|l|}{ Economic coping strategies } \\
\hline \multicolumn{4}{|l|}{ Saving money } \\
\hline \multicolumn{4}{|l|}{ Searching relief materials } \\
\hline \multicolumn{4}{|l|}{ Selecting flood resilient crop variety } \\
\hline \multicolumn{4}{|l|}{ Diversify income sources } \\
\hline \multicolumn{4}{|l|}{ Repairing important damage to the house } \\
\hline \multicolumn{4}{|l|}{ Borrowing money } \\
\hline \multicolumn{4}{|l|}{ Selling important assets } \\
\hline \multirow{2}{*}{\multicolumn{4}{|c|}{$\begin{array}{l}\text { Adjusting consumption } \\
\text { Social coping strategies }\end{array}$}} \\
\hline & & & \\
\hline \multicolumn{4}{|l|}{ Preparing shelter place at friend's or relative's house } \\
\hline \multicolumn{4}{|l|}{ Evacuating family, especially children and elderly to a safe place } \\
\hline \multicolumn{4}{|l|}{ Evacuating the important thing to safe place } \\
\hline \multicolumn{4}{|l|}{ Help other community members in doing work } \\
\hline \multirow{2}{*}{\multicolumn{4}{|c|}{$\begin{array}{l}\text { Preserving food and fuel on the selling of the house } \\
\text { Technological or Structural coping strategies }\end{array}$}} \\
\hline & & & \\
\hline \multicolumn{4}{|l|}{ Getting flood warning by TV, radio or other means of communication } \\
\hline \multicolumn{4}{|l|}{ Building dikes in front of house using sandbags } \\
\hline \multicolumn{4}{|l|}{ Building house with transferable construction materials material } \\
\hline \multicolumn{4}{|l|}{ Increasing the height of the base of the house } \\
\hline Building Machan for saving properties & & & \\
\hline
\end{tabular}

Source: Field Survey, 2012

\section{Local Scale Disaster Risk (LSDR) Model}

The author propose LSDR model as a new conceptualization of local scale disaster risk in relation to climate change. This model is designed to present the relationship between hazard, exposure, vulnerability, resilience and risk. One can apply this model to quantify or assess local scale risk or this model can be readily applied to face trigger events in local scale. This model can be expressed logically as risk is the combination of hazard characteristics, exposure, vulnerability and resilience. Risk can be determined by adding the value of hazard characteristic, exposure, vulnerability and subtracting the value of resilience from the combined value.

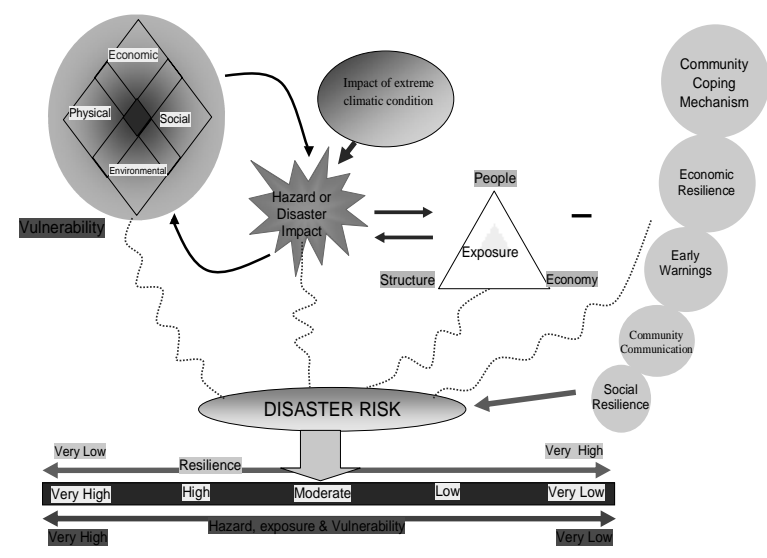

Fig. 7 Local Scale Disaster Risk (LSDR) Model
Several assumptions are implicit in the conceptualization of LSDR. Firstly, this model focuses on local scale disaster risk particularly risk at community level. Secondly, the model was specially created to address natural hazards (e.g. flood), but could be adopted to address man-made hazards (e.g. technological hazard). Thirdly, Hazard and vulnerability interacts to form disaster and finally, while LSDR is a local scale model, individual and community resilience influence disaster risk.

The advantage of this model is that it helps to assess local scale disaster risk by following four factors and associated indicators. Indicators can be changed depending on place and community. Scale of resilience and vulnerability helps to determine risk easily. Although this model is not well fitted for analyzing national scale disaster risk, local scale disaster risk can be assessed properly with this proposed model.

\section{Conclusion}

This study finds that climate change induced flood risk is the combination of hazard, exposure, vulnerability and resilience. The higher the level of resilience the lower is the level of risk and the higher the level of hazard, exposure and vulnerability the higher is the level of risk. The flood problem in the Gangetic char land is closely associated with the rising monsoon rainfall, people and element at risk, physical vulnerability, social vulnerability, economic vulnerability, environmental vulnerability, physical and environmental resilience, social resilience, economic resilience, institutional resilience and community coping mechanism. 


\section{References}

Bollin, C. \& Hidajat, R., Community-based Risk Index: Pilot Implementation in Indonesia, Measuring Vulnerability to Natural Hazards: Towards Disaster Resilient Societies, Jorn Brikmann, United Nations University Press, 2003

Coppola, P. Damon, Introduction to International Disaster Management, Elsevier Inc, 2006

Cutter, S., Boruff, B., \& Shirley, W., Social vulnerability to environmental hazards, Soc. Sci. Quart, 84, 2, 242-260. 2003

DFID, Defining Disaster Resilience: A DFID approach, The Department for International Development, 5-18, 2012

Eakin, H., Institutional change, climate risk, and rural vulnerability: Cases from Central Mexico. World Development, 2005

Haki Z., Akyuerek, Z., and Duezguen, S., Assessment of social vulnerability using geographic information systems: Pendik, Istanbul case study. In: 7th AGILE Conference on Geographic Information Science (Heraklion, Greece, Middle Technical University of Ankara, Turkey) 2004.

IFRC, Vulnerability and Capacity Assessment: An International Federation Guide, Geneva, IFRC, 1999
IPCC.2012, Special Report of the Inter Governmental Panel on Climate Change, ipcc-wg2.gov/SREX/report

Islam, S. N., Singh S., Shaheed H., Wet S., 2010, Settlement Relocations in the char-lands of the Padma River basin in Ganges delta, Bangladesh http://www.academia.edu/1018970/ Settlement_relocations_in_the_char-lands_of_Padma_River_ basin_in_Ganges_delta_Bangladesh

Mujere, N., Flood Frequency Analysis Using The Gumble Distribution, 2011, International Journal on Computer Science and Engineering (IJCSE), http://www.enggjournals. com/ijcse/doc/ IJCSE11-03-07-174.pdf

United Nation Development Programme-Bureau for Crisis Prevention and Recovery (UNDP-BCPR), Reducing Disaster Risk: A Challenge for Development, A Global Report, New York; UNDP Publication, 2004.

UNDP and DFID, Climate Change and Bangladesh, 2012

Manuscript received on 30 October 2013 and revised on 17 November 2013 